\title{
Altered responsiveness to chemokines due to targeted disruption of SHIP
}

\author{
Chang H. Kim, ${ }^{1,2}$ Giao Hangoc, ${ }^{1,2}$ Scott Cooper, ${ }^{1,2}$ Cheryl D. Helgason, ${ }^{3}$ Sandie Yew, ${ }^{3}$ \\ R. Keith Humphries ${ }^{3,4,5}$ Gerald Krystal, $3,5,6$ and Hal E. Broxmeyer ${ }^{1,2}$ \\ ${ }^{1}$ Departments of Microbiology/Immunology and Medicine and the Walther Oncology Center,
Indiana University School of Medicine, Indianapolis, Indiana 46202, USA
${ }^{2}$ Walther Cancer Institute, Indianapolis, Indiana 46208, USA
${ }^{3}$ Terry Fox Laboratory, Vancouver, British Columbia V5Z 1L3, Canada
${ }^{4}$ Department of Pathology,
${ }^{5}$ Department of Laboratory Medicine, and
${ }^{6}$ Department of Medicine, University of British Columbia, Vancouver, British Columbia V5Z 1L3, Canada
}

Address correspondence to: Hal E. Broxmeyer, Department of Microbiology/Immunology and the Walther Oncology Center, Indiana University School of Medicine, Building R4, Room 302, 1044 West Walnut Street, Indianapolis, Indiana 46202-5254, USA. Phone: (317) 274-7510; Fax: (317) 274-7592; E-mail: hbroxmey@iupui.edu.

Chang H. Kim's present address is: Department of Pathology, L235, Stanford University School of Medicine, Stanford, California 94304, USA.

Received for publication May 11, 1999, and accepted in revised form October 26, 1999.

\begin{abstract}
SHIP has been implicated in negative signaling in a number of hematopoietic cell types and is postulated to downregulate phosphatidylinositol-3-kinase-(PI-3K-) initiated events in diverse receptor signaling pathways. Because PI-3K is implicated in chemokine signaling, we investigated whether SHIP plays any role in cellular responses to chemokines. We found that a number of immature and mature hematopoietic cells from SHIP-deficient mice manifested enhanced directional migration (chemotaxis) in response to the chemokines stromal cell-derived factor-1 (SDF-1) and B-lymphocyte chemoattractant (BLC). SHIP $/ /$ - cells were also more active in calcium influx and actin polymerization in response to SDF-1. However, colony formation by SHIP-deficient hematopoietic progenitor cell (HPCs) was not inhibited by 13 myelosuppressive chemokines that normally inhibit proliferation of HPCs. These altered biologic activities of chemokines on SHIP-deficient cells are not caused by simple modulation of chemokine receptor expression in SHIP-deficient mice, implicating SHIP in the modulation of chemokine-induced signaling and downstream effects.
\end{abstract}

J. Clin. Invest. 104:1751-1759 (1999).

\section{Introduction}

Chemokines (chemotactic cytokines) are small ( 10 $\mathrm{kDa})$ proteins capable of inducing diverse effects, including chemotaxis of leukocytes, inhibition of hematopoietic progenitor cell (HPC) proliferation, inhibition or enhancement of angiogenesis, inhibition of HIV infection, and activation and differentiation of leukocytes (1-4). These chemokines specifically or promiscuously bind heterotrimeric G-protein-coupled receptors that have 7 transmembrane domains (5). Engagement of chemokine receptors induces activation of heterotrimeric G-proteins, phospholipase (PL) C, PLD, calcium influx, small $G$ proteins such as RhoA, mitogen-activated protein (MAP) kinases, and phosphatidylinositol 3'-kinases (PI-3-K) and induces phosphorylation of a number of proteins $(6,7)$.

SHIP is a $145-\mathrm{kDa}$ inositol-5-phosphatase containing an $\mathrm{NH}_{2}$-terminal Src homology 2 (SH2) domain, 2 phosphotyrosine binding (PTB) motifs, and a $\mathrm{COOH}$-terminal proline-rich region $(8-10)$. It selectively hydrolyzes in vitro the $5^{\prime}$-phosphate from both inositol 1,3,4,5-tetraphosphate ( $\left.\mathrm{IP}_{4}\right)$ and phosphatidylinositol 3,4,5-triphosphate $\left(\mathrm{PIP}_{3}\right)(8,9)$.
SHIP becomes tyrosine phosphorylated in response to a number of cytokines and associates with Shc (11), the tyrosine phosphatase SHP-2 (12), and in some cell types, Grb2 (13). Recently, it has been shown to inhibit immune receptor activation in both mast cells and $B$ cells by binding to the tyrosine phosphorylated immunoreceptor tyrosine-based inhibition motif (ITIM) of the inhibitory corrector FcyRIIB and by inhibiting FceR1- and B cell receptor-induced calcium influx, respectively (14-17). In addition, SHIP has been shown, even in the absence of FcrRIIB coclustering, to play a "gatekeeper role" in IgE-mediated mast cell degranulation by setting the threshold for and limiting the degranulation process (18). SHIP has also been shown to play an inhibitory role in IL-3- and M-CSF-stimulated growth and survival of hematopoietic cell lines (9, 19). These inhibitory actions of SHIP appear to be attributed, at least in part, to its ability to hydrolyze $\mathrm{PIP}_{3}$, the primary in vivo product of PI- $3 \mathrm{~K}(20)$. By decreasing the levels of PIP $_{3}$, it has also recently been shown to inhibit the activation of protein tyrosine kinase B (PKB)/Akt (21) and Btk (22). 
Mice bearing a targeted disruption of both SHIP alleles have been generated (23). SHIP-null mice are viable and fertile but have a reduced life span. SHIPnull mice demonstrated splenomegaly, extensive consolidation of the lungs due to infiltration by myeloid cells, increased numbers of granulocyte-macrophage progenitors in the bone marrow and spleen, and reduced numbers of bone marrow lymphoid and late erythroid progenitors (CFU-E). HPCs from SHIPnull mice are enhanced in responsiveness to stimulation by cytokines such as GM-CSF, IL-3, steel factor, and M-CSF.

It is known that PI-3K is involved in signaling processes and chemotaxis induced by chemokines including stromal cell-derived factor-1 (SDF-1) (24) and other chemokines (25-27), which suggests a possibility that SHIP plays a role in chemokine-induced biologic effects by antagonizing the effect of PI-3K. We observed that absence of SHIP in mature hematopoietic cells and progenitors resulted in altered cell responses to chemokines. This suggests that SHIP is an important regulator of chemokine responses and signaling.

\section{Methods}

SHIP-deficient mice. Generation of homozygous deletion of SHIP in embryonic stem cells and SHIP-deficient mice has been described previously (23). Sex- and agematched adult mice ( $\sim 6$ weeks old) were used in this study, and wild-type littermates were used as controls.

Cell preparation. Total mouse bone marrow cells were aspirated from 2 femurs of each mouse. Total splenocytes and thymocytes were prepared by mincing spleens or thymus through iron meshes. Lowdensity spleen or thymus mononuclear cells were isolated by centrifuge using Lympholyte-M (Cedarlane, Hornby, Canada). Nonadherent spleen lymphocytes were obtained by removing adherent cells on culture dishes after overnight culture in X-VIVO 20 medium (Biowhittaker Inc., Walkersville, Maryland, USA) supplemented with 20\% FBS (Hyclone Laboratories, Logan, Utah, USA). B220+ spleen B cells were isolated from the low-density splenocytes by staining with biotin-labeled antibodies to CD45R/B220 (clone RA3-6B2, Pharmingen, San Diego, California, USA) followed by positively selecting the stained $\mathrm{B} 220^{+}$ cells using streptavidin-beads and magnets (Miltenyi Biotech, Auburn, California, USA).

Quantitative chemotaxis of HPCs and lenkocytes. Quantitative chemotaxis assays using transwells $(6.5-\mathrm{mm}$ diameter, $5-\mu \mathrm{m}$ pore size, polycarbonate membrane; Costar, Corning, New York, USA.) that allow comparison of chemotaxis of different cells from different mice were performed as described previously $(28$, 29). For chemotaxis of HPC, $5 \times 10^{5}$ bone marrow cells were used for each chemotaxis chamber. SDF-1 (obtained from I. Clark-Lewis, University of British Columbia, Vancouver, Canada) was added to the lower chamber at various concentrations. Chemotaxis was allowed for 4 hours. Input cells and cells migrating to the lower chamber were collected and assayed for colony-forming cells in methylcellulose culture containing various growth factors. Cells from 2 transwells were combined to obtain enough numbers of HPCs for triplicated colony assays. Cells were plated at $5 \times 10^{4}$ per $1 \mathrm{~mL}$ culture medium. Detailed culture conditions are described in the next section. After 7 days, colonies deriving from CFU-GMs were differentially counted using standard techniques under an inverted microscope (30).

For chemotaxis of bone marrow macrophages and B cells, thymocytes, and spleen lymphocytes, a half million low-density mononuclear cells were used for input cells. Chemotaxis was allowed for 3 hours for
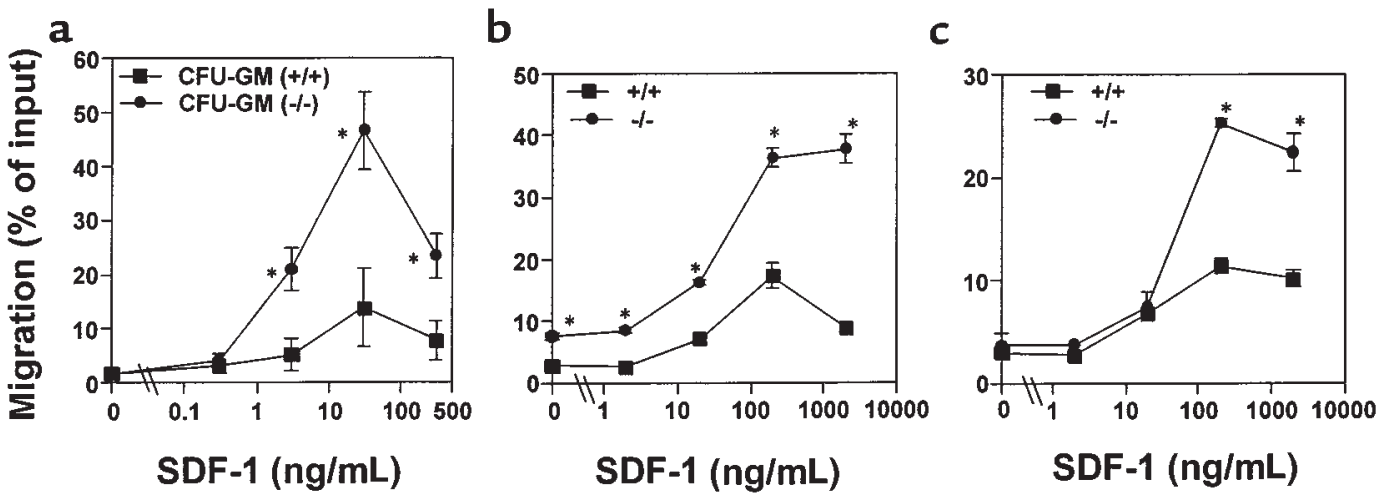

Figure 1

Bone marrow HPC, macrophages and B cells from SHIP-deficient mice have enhanced chemotaxis to SDF-1. (a) Bone marrow cells were examined for their ability to transmigrate from the upper chamber toward SDF-1 at indicated concentrations in the lower chamber. After chemotaxis, myeloid progenitors in input and the lower chamber were assayed by methylcellulose colony assay. HPC (CFU-GM) migration was normalized for the number of input colony-forming HPCs to obtain HPC migration rate (\% of input \pm SD). Chemotaxis of CD11b/Mac$1^{+} \mathrm{F} 4 / 80^{+}$bone marrow macrophages $(\mathbf{b})$ and bone marrow B220+ $\mathrm{B}$ cells $(\mathbf{c})$ in response to SDF- 1 . Data are expressed as the mean $( \pm$ SD) of percent cell migration obtained from triplicated points. Results are each 1 representative of 5 independent experiments with consistent results. ${ }^{*}$ Significant differences were observed between the wild-type and SHIP-deficient cells $(P<0.03)$. 
SDF-1 or 2 hours for murine B-lymphocyte chemoattractant (BLC) and $\mathrm{mCK} \beta-11$ at $37^{\circ} \mathrm{C}$. Input cells and cells migrating to the lower chamber were collected, stained with antibodies (anti-B220-PE, anti-CD11b/Mac-1-TRIcolor, and anti-F4/80-FITC for bone marrow macrophages and $\mathrm{B}$ cells; anti-CD4-Cychrome and anti-CD8-FITC for thymocytes; anti-CD4-Cychrome, anti-B220-PE, and anti-CD8-FITC for splenocytes) and analyzed by FACscan (Becton Dickinson Immunocytometry Systems, Mountain View, California, USA) in a timebased manner for 20 seconds. Anti-CD4-Cychrome (clone L3T4), anti-CD8a-FITC (clone Ly-2), and B220-PE (clone RA3-6B2) were purchased from Pharmingen. Anti-F4/80 was purchased from Serotec Ltd. (Oxford, United Kingdom). Anti-Mac-1 was purchased from Caltag Laboratories (Burlingame, California, USA). Migration of each subset was normalized for the numbers of input cells.

Assays of HPC proliferation. Total bone marrow and spleen cells were plated in the presence or absence of TNF- $\alpha$ or IFN- $\gamma$ each at $10 \mathrm{ng} / \mathrm{mL}$, or $100 \mathrm{ng} / \mathrm{mL}$ of various chemokines (RANTES, fractalkine, MIP-1 $\beta$, MIP-1 $\alpha$, MCP-1, exodus, SLC, TECK, MIP-4, IL-8, IP10, MIG, ENA78, GCP-2, CK $\beta-11$, MCP-4). These cytokine/chemokine concentrations have previously been shown to be optimal for inhibition of colony formations by bone marrow cells from mice of different strains. IFN- $\gamma$, TNF- $\alpha$, and most chemokines were purchased from R\&D Systems Inc. (Minneapolis, Minnesota, USA) except CK $\beta-11$ (31). Bone marrow cells were plated at $5 \times 10^{4}$ per $\mathrm{mL}$ in $1.0 \%$ methylcellulose culture medium with $30 \% \mathrm{vol} / \mathrm{vol}$ FBS (Hyclone) and $1 \mathrm{U} / \mathrm{mL}$ recombinant human erythropoietin (Amgen Biologicals, Thousand Oaks, California, USA), $50 \mathrm{ng} / \mathrm{mL}$ recombinant murine steel factor (Immunex Corp., Seattle, Washington, USA), $5 \%$ vol/vol pokeweed mitogen mouse spleen cell-conditioned medium (30), and $0.1 \mathrm{mM}$ hemin (Kodak Co., Rochester, New York, USA). Colonies deriving from hematopoietic progenitors (CFU-GM, BFU-E, CFU-GEMM) were scored after 7 days of incubation in a humidified environment at $5 \% \mathrm{CO}_{2}$ and lowered (5\%) $\mathrm{O}_{2}$ as described elsewhere (32).

Calcium flux assay. Calcium flux assay in response to chemokines has been described previously (31). Lowdensity nonadherent spleen lymphocytes were used for calcium flux assay. Fluorescence was monitored at 340 and $380 \mathrm{~nm}$ for excitation and $510 \mathrm{~nm}$ for emission for as long as 250 seconds. The data were recorded as the relative ratio of fluorescence excited at 340 and $380 \mathrm{~nm}$. Data were collected every 1 second.

Actin polymerization assay. Low-density thymocytes or splenic nonadherent lymphocytes were used in this assay. Actin polymerization in response to a chemokine has been described previously by others (33). Mean fluorescence was measured by FACscan. $R$ Nase protection assays of chemokine receptor expression. Total RNA was isolated from various sources of cells

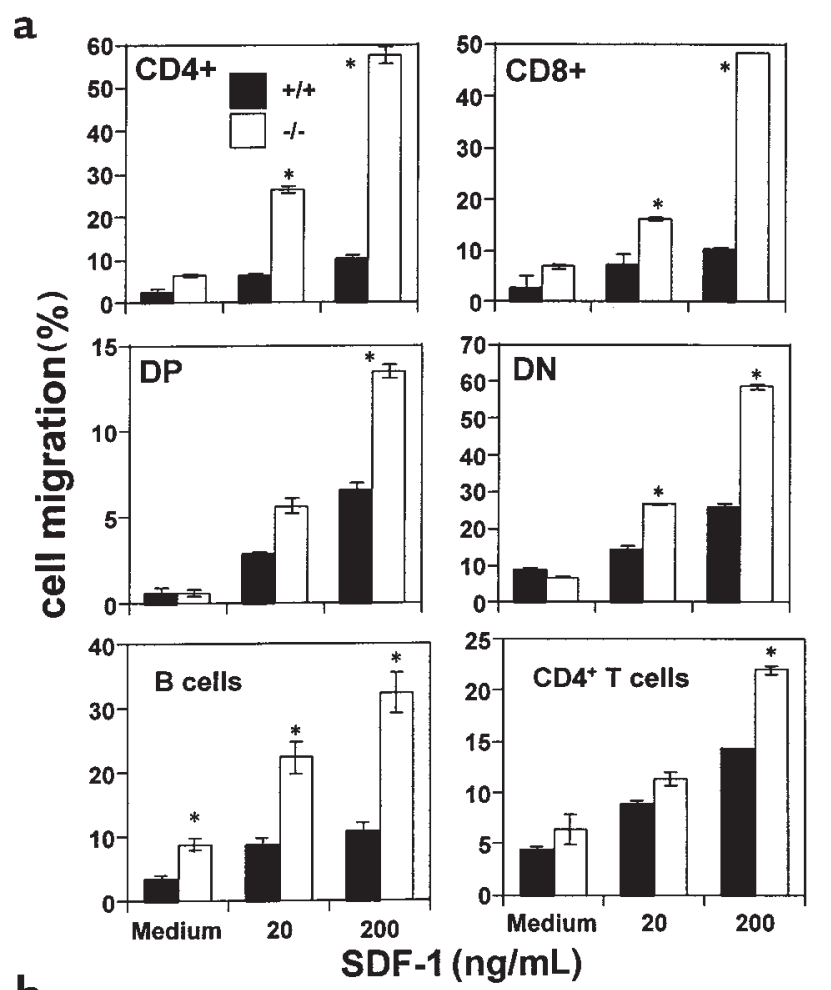

b

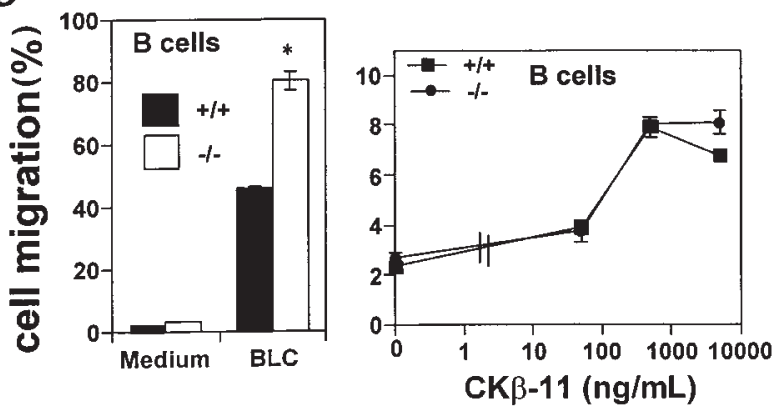

Figure 2

SHIP-deficient hematopoietic cells in thymus and spleen migrate better than wild-type cells to SDF-1 or BLC, but not to CK $\beta-11$. (a) Numbers of cells migrating to the lower chamber containing SDF-1 at indicated concentrations were expressed as percentage of input cells in the upper chamber at the start time of chemotaxis. (b) CK $\beta$ 11 at indicated concentrations $(\mathrm{ng} / \mathrm{mL})$, or BLC at $5 \mu \mathrm{g} / \mathrm{mL}$ was used for chemotaxis of splenic B cells. Data are expressed as the mean ( \pm difference) of percent cell migration obtained from duplicated points. Results are 1 representative of $4-5$ independent experiments. * Significant differences were observed between the wild-type and SHIP-deficient cells $(P<0.05)$.

including bone marrow (total bone marrow cells), thymus (low-density mononuclear cells), and spleen (low-density mononuclear cells and B220 ${ }^{+}$B cells) using Trizol solution (GIBCO BRL/LifeTechnologies, Grand Island, New York, USA). Multiprobe RNase protection template sets mCR5 (CCR1, CCR1b, CCR3, CCR4, CCR5, CCR2, L32, GAPDH) and mCR6 (CXCR2, CXCR4, CXCR5, L32, and GAPDH) were purchased from Pharmingen. RNase protection assay was performed as described previously (34). Filters were dried, exposed on $x$-ray film (X-OMAT; Kodak) 
and analyzed for band radioactivity using phosphorimage analyzer (Molecular Image Analyzer; BioRad Laboratories, Inc., Hercules, California, USA).

Statistical analysis. Student's $t$ test was used to analyze data for significance. $P$ values less than 0.05 were regarded as significant.

\section{Results}

SDF-1 induces greater chemotaxis of SHIP-deficient bone marrow hematopoietic cells than wild-type littermate cells. SDF-1 induces chemotaxis of human HPCs and is constitutively expressed by bone marrow stromal cells $(29,35,36)$. CXCR4 is the receptor for $\operatorname{SDF}-1(37,38)$ and is constitutively expressed on human HPC (37-39). SDF-1 may be an important chemokine that induces homing of HPCs to bone marrow and retains HPCs within the bone marrow. We examined the comparative chemotactic responsiveness of myeloid progenitor cells from SHIP-deficient and wild-type mice. SHIP-deficient CFU-GMs showed significantly enhanced chemotaxis ability in response to SDF-1 (Figure 1a).

Bone marrow macrophages are descendants from bone marrow myeloid progenitors. We examined the chemotactic responsiveness of bone marrow

a
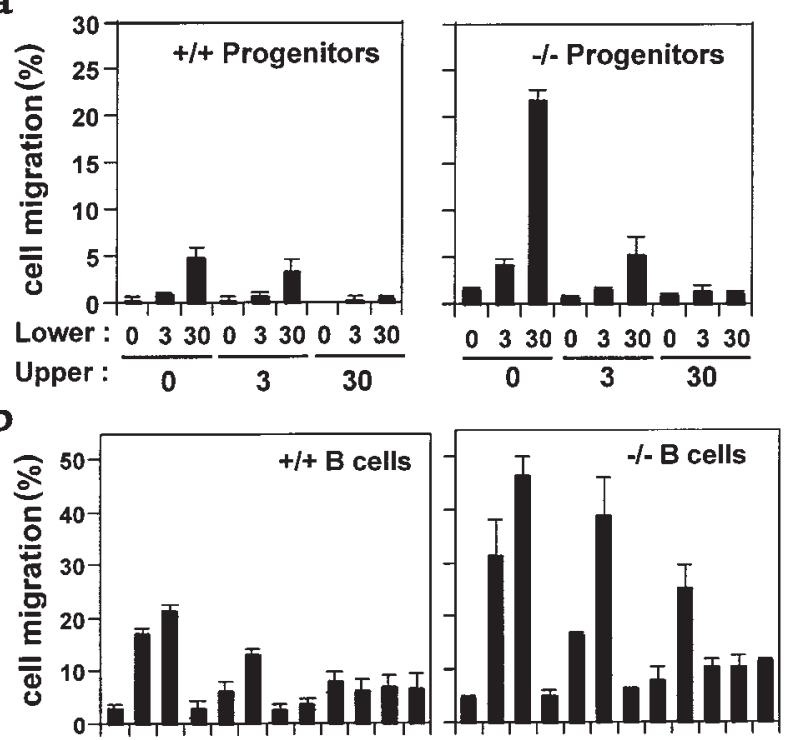

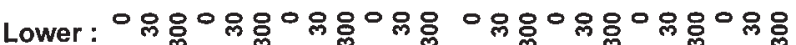

Upper : $\overline{0} \frac{30}{300} \overline{3000} \overline{0} \frac{30}{300} \overline{3000}$ SDF-1 (ng/mL)

\section{Figure 3}

Enhanced migration to SDF-1 is due to enhanced chemotactic, but not chemokinetic, migration. Bone marrow progenitors, CFU-GM (a) and splenic $B$ cells $(\mathbf{b})$ were examined for their chemotactic responsiveness to various concentration gradients of SDF-1 $(\mathrm{ng} / \mathrm{mL})$ in the upper and/or lower chamber in a combinatorial manner. Representative data from 1 of 3 independent experiments is shown in a for CFU-GM, and the combined data of 3 independent experiments is shown for $B$ cells in $\mathbf{b}$ (average $\pm S D$ ). If SD bars are not shown, they were very small. macrophages (CD11b/Mac- $1^{+} \mathrm{F} 4 / 80^{+}$cells) from SHIP-deficient and wild-type mice. SHIP-deficient bone marrow macrophages also showed greater chemotaxis to SDF-1 than their wild-type counterparts (Figure 1b). We also examined the chemotaxis of SHIP-deficient bone marrow lymphoid cells. Bone marrow B cells $\left(B 220^{+}\right)$from SHIP-deficient mice migrated significantly better to SDF-1 than wild-type bone marrow cells (Figure 1c).

Chemotaxis of SHIP-deficient thymic T-cell progenitors and spleen lymphocytes. Thymus expresses several chemokines that show chemotactic activity for thymocyte subsets. Among these chemokines, the chemotactic activity of SDF-1 for thymocytes has been well characterized. SDF-1 attracts 4 thymocyte subsets, immature double-negative (DN: $\left.\mathrm{CD}^{-}{ }^{-} \mathrm{CD} 8^{-}\right)$ and double-positive (DP: $\mathrm{CD} 4^{+} \mathrm{CD} 8^{+}$), and mature single-positive $\left(\mathrm{CD}^{+}\right.$or $\left.\mathrm{CD}^{+}\right)$thymocytes, with a greater preference for immature DN and DP thymocytes (28). We examined whether SHIP-deficient thymocytes manifested a chemotaxis behavior that was different from wild-type thymocytes. We found that SHIP-deficient $\mathrm{CD}^{+}{ }^{+} \mathrm{SP}$ and $\mathrm{CD}^{+}{ }^{+} \mathrm{SP}$ T-cell progenitor subsets, unlike their wild-type counterparts, which are poorly attracted to SDF-1, migrated very well to SDF-1 (Figure 2a). Additionally, the highly responsive subsets of DN and DP thymocytes in wildtype mice were more responsive to SDF-1 in SHIPdeficient mice (Figure 2a).

The chemotactic responsiveness of spleen $\mathrm{B} 220^{+} \mathrm{B}$ cells to SDF-1 was also examined (Figure 2a), as SDF1 is an efficacious chemoattractant for B cells. We observed strikingly enhanced migration of SHIPdeficient B cells to SDF-1. CD4 $4^{+}$spleen T cells from SHIP-deficient mice were also hyper-responsive to SDF-1 (Figure 2a). The difference in chemotaxis to SDF-1 between wild-type and SHIP-deficient $\mathrm{CD}^{+} \mathrm{T}$ cells was less than other cell types such as bone marrow cells and spleen B cells. No significant difference in chemotaxis of SHIP-deficient and wild-type $\mathrm{CD} 8^{+}$ $\mathrm{T}$ cells to SDF-1 was observed (data not shown).

We also addressed the question of whether the enhancement of chemotaxis of SHIP-deficient cells was apparent with other chemokines in addition to SDF-1. We tested 2 other chemokines, BLC/BCA-1 and CK $\beta-11 /$ MIP- $3 \beta /$ ELC, that have comparable chemotactic efficacy for lymphocytes to that of SDF1. BLC attracted more SHIP-deficient B cells than wild-type cells, whereas CK $\beta$ - 11 attracted similar percentages of $\mathrm{B}$ cells from both types of mice (Figure $2 \mathrm{~b})$, demonstrating that SHIP involvement in chemokine-mediated chemotaxis is chemokine specific. Enhanced migration of SHIP-deficient cells to SDF-1 can be due to directional migration (chemotaxis) or random migration (chemokinesis) or both. To address this question, we performed checkerboard chemotaxis assays in the presence of SDF-1 in the lower chamber and/or upper chamber in a combinatorial manner. As SDF-1 concentration in the upper 


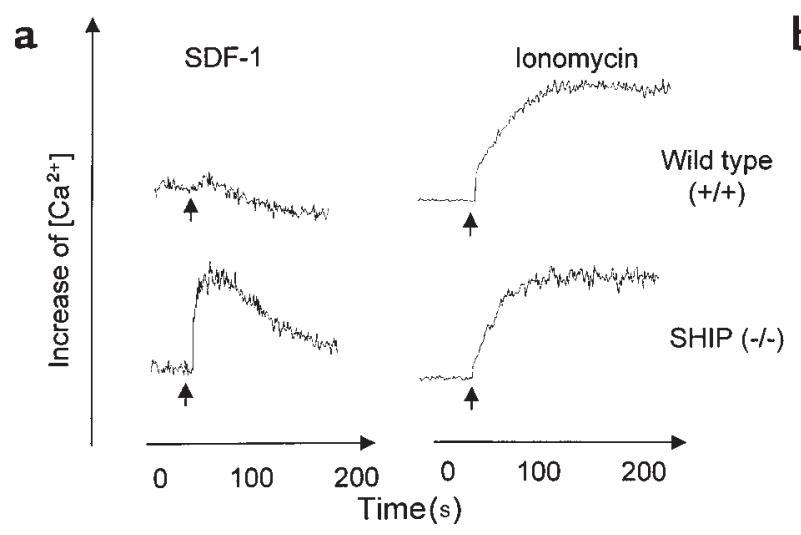

b c
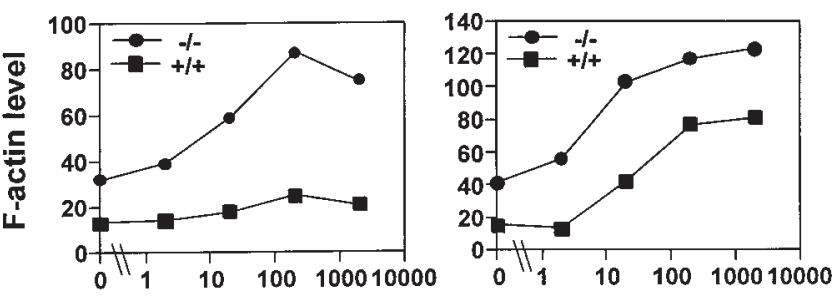

SDF-1 (ng/mL)

\section{Figure 4}

Enhanced biochemical response to CXC chemokine SDF-1. (a) Calcium flux response to SDF-1 in spleen lymphocytes. Optimal doses of SDF$1(100 \mathrm{nM})$ and ionomycin $(0.5 \mu \mathrm{M})$ were used to activate spleen lymphocytes. The $x$-axis and $y$-axis, respectively, represent time in seconds and intracellular calcium concentrations shown as ratio of fluorescence. SDF-1-dependent actin polymerization in splenocytes (b) and thymocytes (c). SDF-1 at indicated concentrations was used to induce actin polymerization. Peak levels of F-actin at 30 seconds after activation were monitored by FACscan after FITC-phalloidin staining of polymerized actin. Results shown are representative of 3 experiments each.

chamber was increased, migration of wild-type and SHIP-deficient HPCs (CFU-GM) and spleen B cells to the lower chamber was decreased (Figure 3). At SDF1 concentrations of $30 \mathrm{ng} / \mathrm{mL}$ for HPCs and 3,000 $\mathrm{ng} / \mathrm{mL}$ for $\mathrm{B}$ cells in the upper chamber, migration of both the wild-type and SHIP-deficient HPCs and B cells toward SDF-1 in the lower chamber was completely decreased to background levels, demonstrating that enhanced motility of both type cells is due to chemotaxis.

Enhanced biochemical response to CXC chemokine SDF-1 in SHIP-deficient cells. Binding of chemokines to their cognate receptors induces G-protein activation, which in turn leads to a calcium influx, presumably as a result of activating protein tyrosine kinases and phospholipase $C \beta$. Although it remains to be elucidated exactly how SHIP inhibits cytokine/antigen/IgEdependent signaling, it has been suggested that it does so, at least in part, by hydrolyzing PIP $\mathrm{P}_{3}$. This reduces the attraction of pleckstrin homology-containing proteins to the plasma membrane, such as PLC $\gamma 1 / 2$ and members of the Tec family, and this leads to a smaller influx of extracellular calcium, either by reducing intracellular calcium release (40) or by inhibiting a critical step between intracellular calcium release and extracellular calcium entry (20). We examined whether chemokine-dependent calcium flux was any different in SHIP-deficient cells and wild-type littermate control cells. Mouse spleen lymphocytes were activated with an optimal dose of SDF1 . The effect of SDF-1 on wild-type murine lymphocytes was barely detectable, as primary resting lymphocytes are poor inducers of calcium flux in response to chemokines (Figure 4a). However, a strong calcium influx was observed in spleen lymphocytes from the SHIP-deficient mice (Figure 4a). On the other hand, wild-type and SHIP-deficient spleen lymphocytes had a similar capacity to increase intracellular calcium concentrations with ionomycin, a calcium ionophore that bypasses cell-surface receptors, which was used as a control for calcium influx. The difference in ability of wild-type and SHIP-deficient spleen cells to mobilize calcium is not due to different compositions of spleen lymphocyte populations from SHIP-deficient and wild-type mice. The composition of the major lymphoid subsets $\left(\mathrm{CD}^{+} \mathrm{T}\right.$ cells and B220 $\mathrm{B}$ cells) that were responsive to SDF-1 in 6-week-old spleen was not much different in SHIPdeficient $\left(14 \% \mathrm{CD}^{+} ; 36 \% \mathrm{~B} 220^{+}\right)$and wild-type $(18 \%$ $\mathrm{CD}^{+} ; 30 \% \mathrm{~B} 220^{+}$) mice.

Transient actin polymerization in response to chemokines is an important cellular event for cell polarization and for formation of cell membrane structures such as uropods and chemotaxis. We examined the F-actin (polymerized actin) levels in SHIP-deficient and wild-type cells before and after chemokine treatment. Splenocytes and thymocytes (Figure 4, b and c, respectively) from SHIP-deficient mice had far greater basal levels of F-actin before activation with SDF-1 than their wild-type counterparts. After activation with SDF-1 at various concentrations, greater F-actin levels were observed in SHIPdeficient versus wild-type thymocytes and spleen lymphocytes (Figure 4, b and c).

SHIP-deficient HPCs are resistant to the suppressive activity of chemokines, but sensitive to that mediated by TNF- $\alpha$ and IFN- $\gamma$. A number of chemokines demonstrate suppressive activity for proliferation of immature subsets of HPCs (41), a biologic activity of many chemokines that is distinct from their chemotactic activity. We tested 13 previously determined myelosuppressive chemokines (MIP-1 $\alpha$, MCP-1, exodus, SLC, TECK, MIP-4, IL-8, IP-10, MIG, ENA78, GCP-2, CK $\beta-11$, and MCP-4), 3 previously determined nonsuppressive chemokines (RANTES, fractalkine, and MIP-1 $\beta$ ), and TNF- $\alpha$ and IFN- $\gamma, 2$ other known sup- 

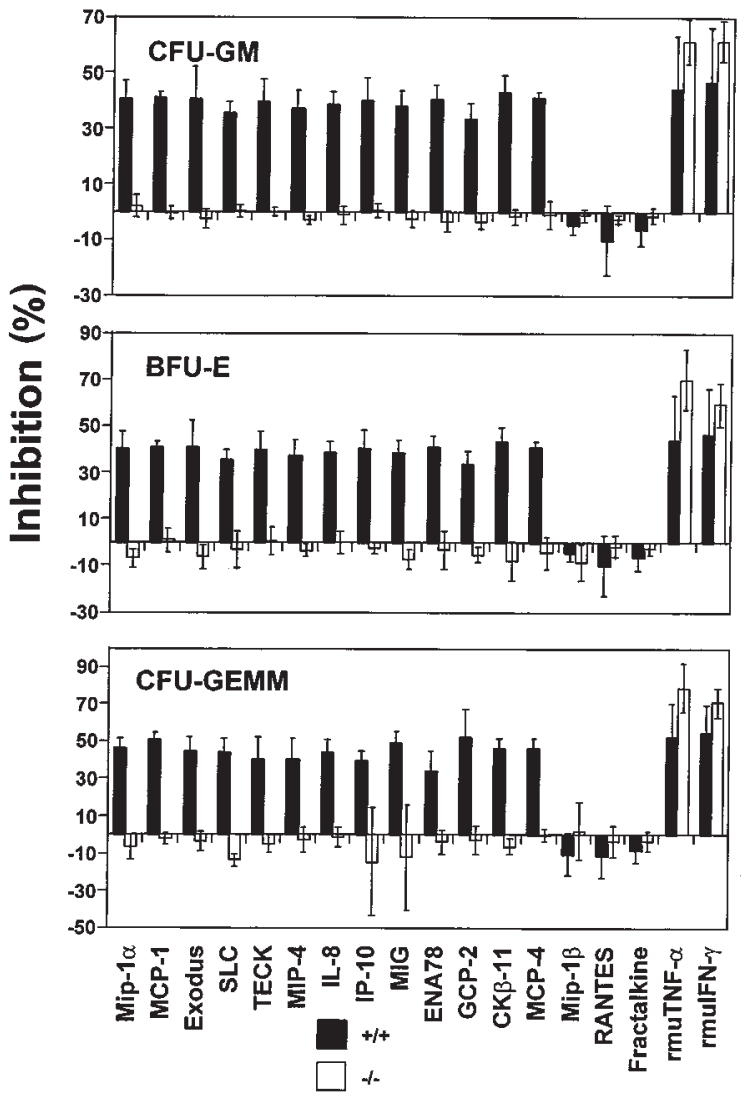

Figure 5

SHIP-deficient HPCs are resistant to the suppressive activity of chemokines but are sensitive to that of TNF- $\alpha$ and IFN- $\gamma$. Bone marrow cells from wild-type and SHIP-deficient mice were plated into methylcellulose culture medium (see Methods) in the presence of PBS, $100 \mathrm{ng} / \mathrm{mL}$ chemokines, or $10 \mathrm{ng} / \mathrm{mL}$ TNF- $\alpha$ and IFN- $\gamma$. Colony formation of HPCs (CFU-GM, BFU-E, CFUGEMM) was assessed after 7 days of incubation. Results shown are the average (mean \pm SD) for 4 separate experiments using wild-type cells and 5 separate experiments using SHIP-deficient cells.

pressive cytokines (42), for their effects on colony formation of bone marrow HPCs. SDF-1 does not suppress the proliferation of immature subsets of HPCs (43). All 13 suppressive chemokines and 2 other cytokines (TNF- $\alpha$ and IFN- $\gamma$ ), but not the 3 nonsuppressive chemokines, inhibited proliferation of wildtype littermate control myeloid progenitor cells (CFU-GM, BFU-E, CFU-GEMM). However, none of the tested chemokines inhibited the proliferation of SHIP-deficient myeloid progenitor cells (Figure 5). In contrast, bone marrow HPCs from SHIP-deficient mice were at least as sensitive as HPCs from wild-type mice to the suppressive effects of TNF- $\alpha$ and IFN- $\gamma$.

Chemokines are active as myelosuppressive cytokines when HPCs are in S-phase of the cell cycle (44). In fact, with normal cells the percent inhibition of colony formation by HPCs in the presence of chemokines is directly related to the percent of HPCs in S-phase. The higher the percent of HPCs in S-phase, the greater the inhibition by chemokines. While SHIP- deficient mice have been reported to have enhanced numbers of CFU-GM, especially in the spleen (23), the proliferative status of these and other subsets of HPCs in marrow was not evaluated. Therefore, we examined the proliferative status of CFU-GM, BFU-E and CFUGEMM (percent in S-phase of the cell cycle as estimated by high specific activity tritiated thymidine kill assay; ref. 30) in the marrow of SHIP-deficient compared with wild-type littermate control mice. CFUGM, BFU-E, and CFU-GEMM in the marrow of SHIPdeficient mice were not reduced in their proliferative capacity compared with these progenitors (data not shown). Thus, the inability of CFU-GM, BFU-E and CFU-GEMM in the marrow of SHIP-deficient mice to respond to inhibition by chemokines could not be attributed to lack of cycling HPC.

Chemokine receptor expression in leukocytes from SHIPdeficient mice and wild-type mice. Altered responsiveness of SHIP-/- hematopoietic cells to chemokines can be a result of changes in the intracellular activation machinery or in chemokine receptor expression. To examine this, we performed RNase protection assays to measure expression of various chemokine receptors in hematopoietic cells from bone marrow, thymus, and spleen. Bone marrow cells from SHIP-deficient mice showed a very similar pattern of chemokine receptor expression as that from wildtype mice (Figure 6). Expression of CXCR4 and CXCR5 was not different in SHIP-deficient spleen cells compared with wild-type cells. SHIP-deficient spleen had enhanced expression of CCR1, CCR2, CCR3, and CXCR2. No difference in expression of chemokine receptors was observed in SHIP-deficient thymocytes compared with wild-type cells. In SHIPdeficient $\mathrm{B} 220^{+} \mathrm{B}$ cells, expression of some CC chemokine receptors including CCR1 and CCR5 was increased over the expression levels in wild-type B cells, whereas expression of CXCR4 and CXCR5 was similar in both types of B cells.

\section{Discussion}

These studies reveal several profound effects of SHIP deficiency on responsiveness to the chemokine SDF1 and other chemokines. On the one hand, the absence of SHIP enhanced chemotaxis, calcium mobilization, and actin polymerization in multiple blood cells and HPCs. Additionally, but in contrast, the absence of SHIP blocked the proliferation inhibition normally associated with a number of chemokines. Our results also suggest that the effects of chemokines on cellular chemotaxis can be dissociated from their effects on progenitor cell proliferation. Specifically, we found that absence of SHIP in spleen lymphocytes makes them far more efficient inducers of calcium influx in response to SDF-1. The detailed signaling events that follow chemokine engagement with its 7-spanner receptor is only just beginning to be revealed. It has been reported that SDF-1 induces activation of CXCR 4 coupled to $\mathrm{G}_{\mathrm{i} 2}$ 
(45), PI-3K, related adhesion focal tyrosine kinase (RAFTK)/pyk2, p44/42 MAP kinases, and NF- $\kappa$ B (26). It has been reported that SDF-1-dependent chemotaxis was inhibited by the selective PI-3K inhibitor, wortmannin. Given that SHIP most likely negatively regulates the resulting $\mathrm{PI}-3 \mathrm{~K}$-induced effects by hydrolyzing $\mathrm{PI} 3,4,5-\mathrm{P}_{3}$ to $\mathrm{PI} 3,4-\mathrm{P}_{2}$, our results suggest that downstream effectors that are activated by elevated PI 3,4,5- $\mathrm{P}_{3}$ levels, such as PKB/Akt (21), Tec (40), and Btk (22) play an important role in chemokine-induced effects. In B lymphocytes, Tec family members have been shown to activate PLC $\gamma$ and enhance calcium influx (40). Thus, it is possible that SHIP downregulates PI-3-K-activated effectors such as Tec. However, the protein intermediates involved in this process still remain to be elucidated. Although chemotactic response of a number of hematopoietic cell types to SDF-1 or BLC was enhanced in SHIP-deficient mice, chemotactic response to another chemokine $C K \beta-11$ was not enhanced. Enhancement of chemotaxis of SHIP-deficient cells versus wild-type cells was greater in thymocytes than splenocytes. This suggests that involvement of SHIP in chemotaxis depends on types of chemokines and on types of cells distinguished by different activation or differentiation states. In this context, it has been reported that the effect of targeted disruption of PI3K is more evident in B cells than in T cells (46).

It was striking to observe that SHIP-deficient HPCs were resistant to the suppressive activity of chemokines on proliferation, as the response of HPCs to SDF-1 in terms of chemotaxis was more enhanced. Suppressive activity of chemokines for proliferation of HPC is a distinct biologic activity from induction of chemotaxis. Among chemokines identified so far, 21 chemokines that cross the $\mathrm{CC}, \mathrm{CXC}$, and $\mathrm{C}$ subfamilies are suppressive, whereas 13 chemokines that cross the CC, $\mathrm{CXC}$, and $\mathrm{CX}_{3} \mathrm{C}$ subfamilies are not (7). However there are only 3 chemokines (SDF-1, SLC, and CK $\beta-11$ ) that have been shown to induce chemotaxis of HPCs (29, $36,43,47)$. The resistance of SHIP-deficient HPCs to the suppressive chemokines is reminiscent of the resistance of HPCs from patients with leukemia $(48,49)$. It would be interesting to examine whether SHIP and related signaling pathways are intact in these leukemic HPCs. SHIP-deficient HPCs, in contrast, are at least as sensitive to the suppressive effects of 2 other inhibitory cytokines (TNF- $\alpha$ and IFN- $\gamma$ ), implying that the mechanism of suppression by chemokines is different from that of nonchemokine inhibitory cytokines.

Altered response to chemokines can be caused by altered expression of chemokine receptors, which is often observed during differentiation and activation of various hematopoietic cell types. We examined the possibility of altered expression of chemokine receptors in SHIP-deficient leukocytes by RNase protection assay. Bone marrow cells from wild-type and SHIPdeficient mice did not differ in their expression profile of chemokine receptors for CCR1, CCR2, CCR3, CCR5, CXCR2, and CXCR5. Among these receptors, CCR2 and CXCR2, respectively, have previously been demonstrated using receptor knockout mice to be dominant receptors for inhibition of progenitor cell proliferation by MCP-1 (and MCP-4; refs. 2, 50, and 51), and IL-8 (and ENA-78, GCP-2; refs. 2 and 52). Using marrow cells from CCR1 (-/-) mice, CCR1 was shown not to be a major receptor of MIP- $1 \alpha$ for inhibition of progenitor proliferation (53). Therefore, CCR5, another receptor for MIP- $1 \alpha$, may be a major receptor for this biologic activity of MIP-1 $\alpha$. Thus, it is not likely that the resistance of SHIP-deficient HPCs to the suppressive chemokines such as MCP-1, MCP-4, IL-8, ENA-78, GCP-2, and MIP-1 $\alpha$ is caused by loss of chemokine receptor expression. Expression of chemokine receptors examined in thymocytes from SHIP-deficient mice showed no difference from those of wild-type mice. However, some chemokine receptors in SHIP-deficient splenic leukocytes had different expression patterns from wild-type cells, whereas expression of other receptors remained the same. Expression of CXCR4 and CXCR5, the receptors for SDF-1 and BLC, respectively, are relatively similar in wild-type and SHIP-deficient mice. Thus, we believe

\section{Figure 6}

Chemokine receptor expression in leukocytes from SHIP-deficient $(-)$ and wild-type $(+)$ mice. Freshly isolated bone marrow cells $(B M)$, spleen mononuclear cells (Spln), thymocytes (thym), and B220+ B cells (B cells; purity $>95 \%$ ) from wild-type and SHIP-deficient mice were analyzed for expression of chemokine receptor mRNA by RNase protection assay. In addition to the chemokine receptors, L32 and GAPDH were included as internal controls. Expression of CC chemokine receptors is shown in $\mathbf{a}$, and that of CXC chemokine receptors is shown in $\mathbf{b}$. These results are representative of 3-4 reproducible experiments.
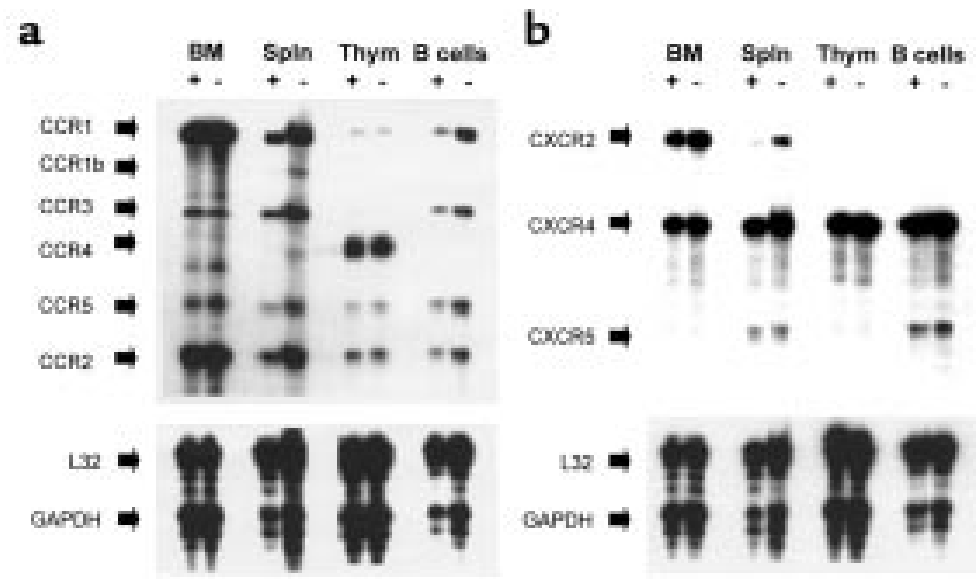
that the enhanced effect of these chemokines is not due to enhanced chemokine receptor expression.

Some of the pathological phenotypes of SHIP-deficient mice may be explained by the modulation of chemokine effects. SHIP-deficient mice have increased numbers of myeloid cells in bone marrow and spleen (23). Reasons for this may include enhanced progenitor cell proliferation due in part, perhaps, to insensitivity of these early cells to suppression by the chemokines, which can be found in marrow, spleen, blood, and other organs. Additionally, the enhanced chemotaxis of progenitor cells in response to SDF-1 may be responsible for the greater attraction and/or retention of these cells in the marrow and spleen.

\section{Acknowledgments}

This work was supported by grants from the U.S. Public Health Service (RO1 DK 53674, R01 HL 56416, and R01 HL 54037), by a project of the National Institutes of Health (P01 HL 53586) to H.E. Broxmeyer, and by the National Cancer Institute-Canada (NCIC) and the Medical Research Council-Canada, with core support from the British Columbia Cancer Foundation and the British Columbia Cancer Agency. C.H. Kim is a Fellow of the Leukemia Society of America. S. Yew holds a fellowship from the National Sciences and Engineering Research Council of Canada, and G. Krystal is a Terry Fox Cancer Research Scientist of the NCI-C, supported by funds from the Canada Cancer Society and Terry Fox Run.

1. Baggiolini, M., Dewald, B., and Moser, B. 1997. Human chemokines: an update. Ann. Rev. Immunol. 15:675-705.

2. Broxmeyer, H.E., and Kim, C.H. 1999. Regulation of hematopoiesis in a sea of chemokine family members with a plethora of redundant activities (review). Exp. Hematol. 27:1113-1123.

3. Kim, C.H., and Broxmeyer, H.E. 1999. Chemokines: signal lamps for trafficking of T-and B-cells for development and effector function. $J$. Leukoc. Biol. 65:6-15.

4. Strieter, R.M., Polverini, P.J., Arenberg, D.A., and Kunkel, S.L. 1995. The role of CXC chemokines as regulators of angiogenesis. Shock. 4:155-160.

5. Murphy, P.M. 1996. Chemokine receptors: structure, function and role in microbial pathogenesis. Cytokine Growth Factor Rev. 7:47-64.

6. Ward, S.G., Bacon, K., and Westwick, J. 1998. Chemokines and T lymphocytes: more than an attraction. Immunity. 9:1-11.

7. Kim, C.H., and Broxmeyer, H.E. 1999. Chemokines for immature blood cells: effects on migration, proliferation and differentiation. In Chemokines and diseases. M.E. Rothenberg, editor. Marcel Dekker. New York, NY. 227-261.

8. Damen, J.E., et al. 1996. The $145-\mathrm{kDa}$ protein induced to associate with Shc by multiple cytokines is an inositol tetraphosphate and phosphatidylinositol 3,4,5- triphosphate 5-phosphatase. Proc. Natl. Acad. Sci. USA. 93:1689-1693.

9. Lioubin, M.N., et al. 1996. p150Ship, a signal transduction molecule with inositol polyphosphate-5- phosphatase activity. Genes Dev. 10:1084-1095

10. Kavanaugh, W.M., et al. 1996. Multiple forms of an inositol polyphosphate 5-phosphatase form signaling complexes with Shc and Grb2. Curr. Biol. 6:438-445.

11. Liu, L., et al. 1997. The Src homology 2 (SH2) domain of SH2-containing inositol phosphatase (SHIP) is essential for tyrosine phosphorylation of SHIP, its association with Shc, and its induction of apoptosis. J. Biol. Chem. 272:8983-8988.

12. Sattler, M., et al. 1997. The phosphatidylinositol polyphosphate 5phosphatase SHIP and the protein tyrosine phosphatase SHP-2 form a complex in hematopoietic cells which can be regulated by BCR/ABL and growth factors. Oncogene. 15:2379-2384.
13. Osborne, M.A., et al. 1996. The inositol 5' -phosphatase SHIP binds to immunoreceptor signaling motifs and responds to high affinity $\operatorname{IgE}$ receptor aggregation. J. Biol. Chem. 271:29271-29278.

14. Ono, M., Bolland, S., Tempst, P., and Ravetch, J.V. 1996. Role of the inositol phosphatase SHIP in negative regulation of the immune system by the receptor Fc(gamma)RIIB. Nature. 383:263-266.

15. Ono, M., et al. 1997. Deletion of SHIP or SHP-1 reveals two distinct pathways for inhibitory signaling. Cell. 90:293-301.

16. Liu, Q., et al. 1998. The inositol polyphosphate 5-phosphatase ship is a crucial negative regulator of B cell antigen receptor signaling. J. Exp. Med. 188:1333-1342.

17. Kimura, T., Sakamoto, H., Appella, E., and Siraganian, R.P. 1997. The negative signaling molecule $\mathrm{SH} 2$ domain-containing inositolpolyphosphate 5-phosphatase (SHIP) binds to the tyrosine-phosphorylated beta subunit of the high affinity IgE receptor. J. Biol. Chem. 272:13991-13996.

18. Huber, M., et al. 1998. The src homology 2-containing inositol phosphatase (SHIP) is the gatekeeper of mast cell degranulation. Proc. Natl. Acad. Sci. USA. 95:11330-11335.

19. Liu, L., Damen, J.E., Ware, M.D., and Krystal, G. 1997. Interleukin-3 induces the association of the inositol 5-phosphatase SHIP with SHP2. J. Biol. Chem. 272:10998-11001.

20. Huber, M., et al. 1998. Targeted disruption of SHIP leads to Steel fac tor-induced degranulation of mast cells. EMBO J. 17:7311-7319.

21. Aman, M.J., Lamkin, T.D., Okada, H., Kurosaki, T., and Ravichandran, K.S. 1998. The inositol phosphatase SHIP inhibits Akt/PKB activation in B cells. J. Biol. Chem. 273:33922-33928.

22. Bolland, S., Pearse, R.N., Kurosaki, T., and Ravetch, J.V. 1998. SHIP modulates immune receptor responses by regulating membrane association of Btk. Immunity. 8:509-516.

23. Helgason, C.D., et al. 1998. Targeted disruption of SHIP leads to hemopoietic perturbations, lung pathology, and a shortened life span. Genes Dev. 12:1610-1620.

24. Ganju, R.K., et al. 1998. The alpha-chemokine, stromal cell-derived factor-1alpha, binds to the transmembrane G-protein-coupled CXCR-4 receptor and activates multiple signal transduction pathways. J. Biol. Chem. 273:23169-23175.

25. Knall, C., Worthen, G.S., and Johnson, G.L. 1997. Interleukin 8-stimulated phosphatidylinositol-3-kinase activity regulates the migration of human neutrophils independent of extracellular signal-regulated kinase and p38 mitogen-activated protein kinases. Proc. Natl. Acad. Sci. USA. 94:3052-3057.

26. Turner, L., Ward, S.G., and Westwick, J. 1995. RANTES-activated human $\mathrm{T}$ lymphocytes. A role for phosphoinositide 3- kinase. $J$. Immunol. 155:2437-2444.

27. Turner, S.J., Domin, J., Waterfield, M.D., Ward, S.G., and Westwick, J. 1998. The CC chemokine monocyte chemotactic peptide-1 activates both the class I p85/p110 phosphatidylinositol 3-kinase and the class II PI3K- C2alpha. J. Biol. Chem. 273:25987-25995.

28. Kim, C.H., Pelus, L.M., White, J.R., and Broxmeyer, H.E. 1988. Differential chemotactic behavior of developing $\mathrm{T}$ cells in response to thymic chemokines. Blood. 91:4434-4443.

29. Kim, C.H., and Broxmeyer, H.E. 1998. In vitro behavior of hematopoietic progenitor cells under the influence of chemoattractants: SDF-1, steel factor and the bone marrow environment. Blood. 91:100-110.

30. Cooper, S., and Broxmeyer, H.E. 1996. Measurement of interleukin-3 and other hematopoietic growth factors, such as GM-CSF, G-CSF, M$\mathrm{CSF}$, and erythropoietin and the potent co-stimulating cytokines steel factor and Flt3 ligand. In Current protocols in immunology. Coligan, J.E., et al., editors. John Wiley \& Sons Inc. New York, NY. Suppl. 18 6.4.1-6.4.12.

31. Kim, C.H., et al. 1998. CK beta-11/macrophage inflammatory protein3 beta/EBI1- ligand chemokine is an efficacious chemoattractant for $\mathrm{T}$ and B cells. J. Immunol. 160:2418-2424.

32. Broxmeyer, H.E., et al. 1990. Enhancing and suppressing effects of recombinant murine macrophage inflammatory proteins on colony formation in vitro by bone marrow myeloid progenitor cells. Blood. 76:1110-1116.

33. Howard, T.H., and Meyer, W.H. 1984. Chemotactic peptide modulation of actin assembly and locomotion. J. Cell. Biol. 98:1265-1271.

34. Kim, C.H., et al. 1999. Abnormal chemokine-induced responses of immature and mature hematopoietic cells from motheaten mice implicate the protein tyrosine phosphatase SHP-1 in chemokine responses. J. Exp. Med. 190:681-690.

35. Bleul, C.C., Fuhlbrigge, R.C., Casasnovas, J.M., Aiuti, A., and Springer T.A. 1996. A highly efficacious lymphocyte chemoattractant, stromal cell-derived factor 1 (SDF-1). J. Exp. Med. 184:1101-1109.

36. Aiuti, A., Webb, I.J., Bleul, C., Springer, T., and Gutierrez Ramos, J.C. 1997. The chemokine SDF-1 is a chemoattractant for human CD34+ hematopoietic progenitor cells and provides a new mechanism to explain the mobilization of CD34+ progenitors to peripheral blood. J. Exp. Med. 185:111-120. 
37. Bleul, C.C., et al. 1996. The lymphocyte chemoattractant SDF-1 is a ligand for LESTR/ fusin and blocks HIV-1 entry. Nature. 382:829-833.

38. Oberlin, E., et al. 1996. The CXC chemokine SDF-1 is the ligand for LESTR/fusin and prevents infection by T-cell-line-adapted HIV-1. Nature. 382:833-835.

39. Mohle, R., et al. 1998. The chemokine receptor CXCR-4 is expressed on CD34+ hematopoietic progenitors and leukemic cells and mediates transendothelial migration induced by stromal cell-derived factor- 1 . Blood. 91:4523-4530.

40. Scharenberg, A.M., et al. 1998. Phosphatidylinositol-3,4,5-trisphosphate (PtdIns-3,4,5-P3)/Tec kinase-dependent calcium signaling pathway: a target for SHIP-mediated inhibitory signals. EMBO J. 17:1961-1972.

41. Broxmeyer, H.E., et al. 1999. Effects of CC, CXC, C and CX3C chemokines on proliferation of myeloid progenitor cells, and insights into SDF-1 induced chemotaxis of progenitors. Ann. NY Acad. Sci. 872:142-163.

42. Broxmeyer, H.E. 1996. Myelosuppressive cytokines and peptides. In: Blood cell biochemistry, vol. 7. T. Whetton, and T. Gordon, editors. Plenum Press. London, United Kingdom. 121-150.

43. Kim, C.H., and Broxmeyer, H.E. 1999. SLC/exodus2/6Ckine/TCA4 induces chemotaxis of hematopoietic progenitor cells: differential activity of ligands of CCR7, CXCR3, or CXCR4 in chemotaxis vs. suppression of progenitor proliferation. J. Leukoc. Biol. 66:455-461.

44. Mantel, C., Kim, Y.J., Cooper, S., Kwon, B., and Broxmeyer, H.E. 1993. Polymerization of murine macrophage inflammatory protein 1 alpha inactivates its myelosuppressive effects in vitro: the active form is a monomer. Proc. Natl. Acad. Sci. USA. 90:2232-2236.

45. Moepps, B., Frodl, R., Rodewald, H.R., Baggiolini, M., and Gierschik, P. 1997. Two murine homologues of the human chemokine receptor
CXCR4 mediating stromal cell-derived factor 1alpha activation of Gi2 are differentially expressed in vivo. Eur. J. Immunol. 27:2102-2112.

46. Fruman, D.A, et al. 1999. Impaired B cell development and proliferation in absence of phosphoinositide 3-kinase p85alpha. Science. 283:393-397.

47. Kim, C.H., Pelus, L.M., White, J.R., and Broxmeyer, H.E. 1998. MIP-3 beta/ELC/CKb-11 is a chemoattractant with a specificity for macrophage progenitors among myeloid progenitor cells. J. Immunol. 161:2580-2585.

48. Eaves, C.J., Cashman, J.D., Wolpe, S.D., and Eaves, A.C. 1993. Unresponsiveness of primitive chronic myeloid leukemia cells to macrophage inflammatory protein 1 alpha, an inhibitor of primitive normal hematopoietic cells. Proc. Natl. Acad. Sci. USA. 90:12015-12019.

49. Broxmeyer, H.E., et al. 1995. Human chemokines: enhancement of specific activity and effects in vitro on normal and leukemic progenitors and a factor-dependent cell line and in vivo in mice. Ann. Hematol. 71:235-246.

50. Boring, L., et al. 1997. Impaired monocyte migration and reduced type 1 (Th1) cytokine responses in C-C chemokine receptor 2 knockout mice. J. Clin. Invest. 100:2552-2561.

51. Reid, S., et al. 1999. Enhanced myeloid progenitor cell cycling and apoptosis in mice lacking the chemokine receptor, CCR2. Blood. 93:1524-1533.

52. Broxmeyer, H.E., et al. 1996. Interleukin-8 receptor is involved in negative regulation of myeloid progenitor cells in vivo: evidence from mice lacking the murine IL-8 receptor homolog. J. Exp. Med. 184:1825-1832.

53. Broxmeyer, H.E., Cooper, S., Hangoc, G., Gao, J.L., and Murphy, P.M. 1999. Dominant myelopoietic effector functions mediated by chemokine receptor CCR1. J. Exp. Med. 189:1987-1992. 